\title{
163. IPSP-Receptive Field in the Cerebellum for Deiters' Neurones
}

\author{
By Masao Iтo and Nobufumi KawaI \\ Department of Physiology and Department of Neuropsychiatry, \\ Faculty of Medicine, University of Tokyo \\ (Comm. by Yas Kuno, M.J.A., Nov. 12, 1964)
}

It was reported previously ${ }^{1)}$ that, when the cerebellar cortex was stimulated at the anterior vermian part, inhibitory postsynaptic potentials (IPSPs) were produced monosynaptically in Deiters' neurones on the homolateral side. When considered in conjunction with the well-known inhibitory action which the cerebellum exerts upon the bulbar postural centres, it has been suggested that these monosynaptic IPSPs were mediated by the cerebellar corticofugal fibres. A question would arise: Is there really a regional correspondence between the sites giving rise to the IPSPs and the areas involving those cerebellar Purkinje cells which project directly onto Deiters' neurones? This has now been answered in the experiments to be reported by using the multipolar cerebellar electrodes.

Cats were anaesthetized with Nembutal. The dissection and experimental procedure were already described. ${ }^{2)}$ The multipolar electrodes were made by sticking five pieces of enamel wire (diameter, $0.1 \mathrm{~mm}$ ) to a shaft of steel wire (diameter, $0.15 \mathrm{~mm}$ ), their cut ends being distributed along the shaft at intervals of 2 or $3 \mathrm{~mm}$ (Fig. A). One electrode thus provided five poles on a straight line at these intervals. In the case of Fig. B, four electrodes of this type were mounted on a micromanipulator in parallel with each other at intervals of $2 \mathrm{~mm}$ and were inserted over the posterior lobe into the culmen (a in Fig. B). Their depth from the surface of the culmen was at the most $3 \mathrm{~mm}$. The spacing of totally twenty stimulating poles on and under the cerebellar surface is shown in Fig. C; they were placed in the anterior lobe except for the caudal four (Nos. 1, 6, 11, 16) which were located on the posterior lobe. Stimulation was done by applying brief square pulses (duration, 0.08 to $0.2 \mathrm{msec}$ ) in turn between the two neighbouring poles on each electrode. Currents were passed in the caudo-cranial direction except when the most caudal poles were used as cathode.

In the cat of Figs. B and C six Deiters' neurones were impaled at the same cranio-caudal level of the nucleus and therefrom potential changes were recorded intracellularly by stimulating each of the cerebellar spots with pulses of 10 volts. Fig. D exemplifies an IPSP 


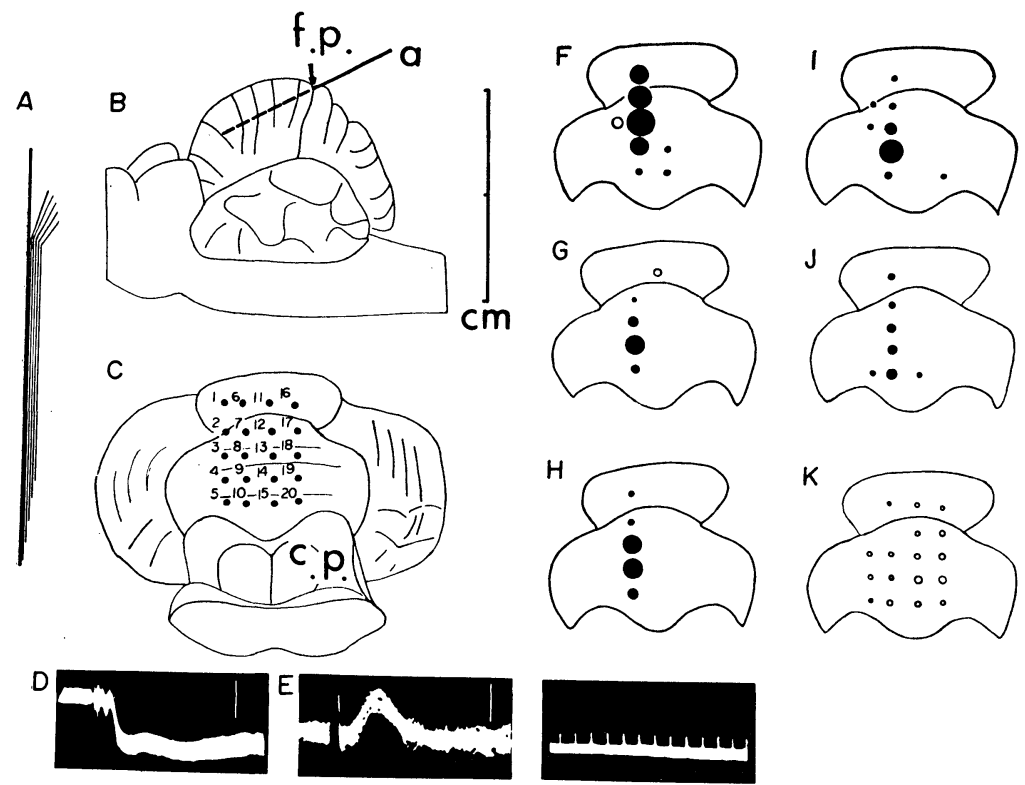

Figs. A-K. A: Multipolar cerebellar electrode. B: Side view of the cerebellum showing the direction (a) along which the multipolar electrodes were inserted into the culmen. f.p., fissura prima. C: Cranio-dorsal view of the cerebellum illustrating the spacing of the poles Nos. 1 to 20. c.p., colliculi posteriores. D: Example of the cerebellar-evoked IPSPs in a Deiters' neurone. E: That of the EPSPs in another cell. Time scale; msec. Voltage calibration; $5 \mathrm{mV}$ in D and $2 \mathrm{mV}$ in E. F-K: Relative amplitudes of the monosynaptic PSPs are indicated by sizes of the circles (• for IPSPs and $\circ$ for EPSPs) in the outlines of the anterior and posterior lobes, at positions corresponding to the stimulating spots of $\mathrm{C}$.

which occurred in one of them monosynaptically from the spot No. 8. For each neurone the amplitudes of the monosynaptic IPSPs were measured at their summit and their relative values were indicated in Figs. $\mathrm{E}$ to $\mathrm{J}$ by the sizes of the closed circles which were drawn on their respective sites of initiation. It is pointed out that, though the spatial pattern of the IPSP-field varies cell by cell, there is always a remarkable predominance in the homolateral vermian part. The general feature of the IPSP field remained the same even when the stimulus intensity for the survey was increased up to 30 volts. Similar tests were successfully made in six cats on seventy-eight Deiters' neurones and revealed an invariable dominance of the homolateral anterior vermis in producing the monosynaptic IPSPs. In the cases where the stimulating electrodes were partly placed in the lobules VI and VII of the posterior lobe, it was found that about half of the examined cells received IPSPs monosynaptically from the homolateral vermis throughout the posterior and anterior lobes. In the lobules V, VI and VII, when tested with pulses of 10 volts, the medial 
border of the receptive field never extended over the mid sagittal line, while in the lobule IV or III it often covered the contralateral vermian part (Figs. F, I, J). Its lateral extension also varied case by case and it often spread over the line indicated by the paravermian vein (Figs. I, J).

The above described localization of the IPSP-receptive field is in good agreement with the histologically determined regional distribution of the cerebellar projection onto Deiters' neurones: Dominantly on the homolateral anterior vermis and less prominently on the homolateral posterior vermis. ${ }^{3)}$ Spatial correspondence has also been indicated between the intracerebellar nuclei and the cerebellar cortex which inhibits the former, ${ }^{4}$ in accordance with the histological scheme of the cerebellar efferent projections; from the anterior lobe to the homolateral nuclei fastigii and interpositus and from the hemisphere to the homolateral dentate nucleus. ${ }^{5)}$ These results strongly support the view that the cerebellar Purkinje cells are inhibitory and so produce IPSPs in their target neurones. ${ }^{1)}$

Excitatory postsynaptic potentials (EPSPs) were noticed often to occur in Deiters' neurones under stimulation outside the receptive field for IPSPs, as exemplified in Fig. E and indicated in F and $G$ by open circles. They occurred with latency of 0.6 to $1.0 \mathrm{msec}$ and hence are presumed to be produced monosynaptically. In about $15 \%$ of the examined Deiters' neurones it happened that the EPSP-receptive field covered the almost whole area of the culmen as well as of the posterior lobe, either homolateral or contralateral, as indicated in Fig. K. In respect of this wide extension of the receptive field, it is unlikely that these EPSPs were caused by the cerebellar corticofugal fibres which in those cases might have been projected from the other regions of the anterior lobe. A possible explanation would then be that some cerebellar afferent fibres take the nucleus of Deiters and the cerebellar cortex as their common targets and therefore that excitation at the latter makes impulses to fire back into the former. Primary vestibular fibres, dorsal spino-cerebellar fibres and the olivo-cerebellar fibres would have such synaptic connexions, ${ }^{2,3), 63}$ which, however, have yet to be investigated.

\section{References}

1) Ito, M., and Yoshida, M.: Experientia, 20, 515 (1964).

2) Ito, M., Hongo, T., Yoshida, M., Okada, Y., and Obata, K.: Experientia, 20, 295 (1964).

3) Brodal, A., Pompeiano, O., and Walberg, F.: The Vestibular Nuclei and their Connections. C. C. Thomas, Springfield, Ill., pp. 55-59, 64-72 (1962).

4) Ito, M., Yoshida, M., and Obata, K.: Experientia, 20, 575 (1964).

5) Jansen, J.: In Aspect of Cerebellar Anatomy, ed. by J. Jansen and A. Brodal, Johan Grundt Tanum Forlag, Oslo, pp. 189-248 (1954).

6) Brodal, A.: Ibid., pp. 82-188. 